\title{
Role of Cooperative Interactions in the Intercalation of Heteroatoms between Graphene and a Metal Substrate
}

Geng Li, ${ }^{\dagger}, \#$ Haitao Zhou, ${ }^{\dagger, \#}$ Lida Pan, ${ }^{\dagger, \#}$ Yi Zhang, ${ }^{\dagger}$ Li Huang, $^{\dagger}$ Wenyan Xu, ${ }^{\dagger}$ Shixuan Du, ${ }^{\dagger}$ Min Ouyang, Andrea C. Ferrari, ${ }^{*} \S$ and Hong-Jun Gao ${ }^{* \dagger}$

${ }^{\dagger}$ Institute of Physics, Chinese Academy of Sciences, Beijing 100190, P.R. China

${ }^{\ddagger}$ Department of Physics and Center for Nanophysics and Advanced Materials, University of Maryland, College Park, Maryland 20742, United States

${ }^{\S}$ Cambridge Graphene Centre, University of Cambridge, Cambridge, CB3 0FA, United Kingdom

Supporting Information

ABSTRACT: The intercalation of heteroatoms between graphene and a metal substrate has been studied intensively over the past few years, due to its effect on the graphene properties, and as a method to create vertical heterostructures. Various intercalation processes have been reported with different combinations of heteroatoms and substrates. Here we study $\mathrm{Si}$ intercalation between graphene and $\mathrm{Ru}(0001)$. We elucidate the role of cooperative interactions between heteroatoms, graphene, and substrate. By combining scanning tunneling microscopy with density functional theory, the

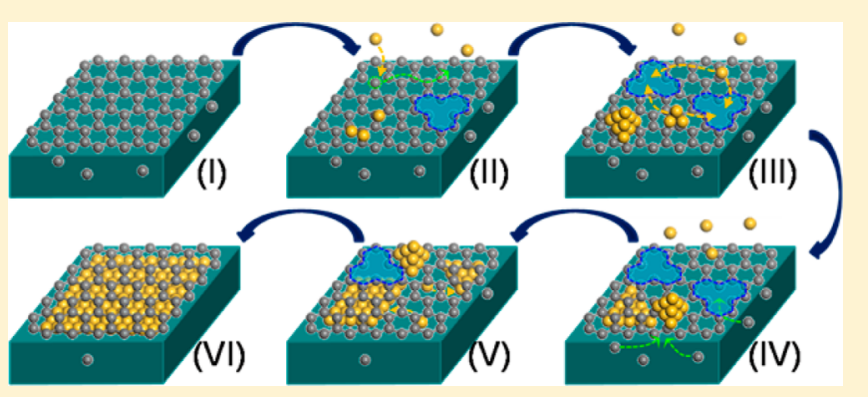
intercalation process is confirmed to consist of four key steps, involving creation of defects, migration of heteroatoms, self-repairing of graphene, and growth of an intercalated monolayer. Both theory and experiments indicate that this mechanism applies also to other combinations of hetero-atoms and substrates.

\section{INTRODUCTION}

Graphene, a two-dimensional crystal of carbon atoms packed in a honeycomb structure, has many promising mechanical, ${ }^{1}$ electrical, ${ }^{1-3}$ and optical ${ }^{1,4}$ properties. ${ }^{1}$ Graphene can also interact with various heteroatoms or molecules, resulting in a change of its intrinsic properties. This can also be used to create hybrid graphene-based heterostructures, ${ }^{1,5-18}$ offering a new degree of freedom to design functional graphene-based device architectures. A variety of atomic ${ }^{5-13}$ and molecular ${ }^{14,15,17}$ species have been successfully integrated with graphene on substrates via intercalation. Understanding this process may be critical in realizing novel graphene-based heterostructures. ${ }^{19,20}$

Here we combine atomic-scale characterization with density functional theory (DFT) and investigate the key processes governing the intercalation of heteroatoms between graphene and a substrate. Si intercalation between graphene and $\mathrm{Ru}(0001)$ is chosen as a test bed because: (1) Si plays an important role in electronics, and the development of hybrid graphene-Si structures/devices may offer a seamless integration route with current microelectronics processes. (2) Graphene can be routinely grown on $\mathrm{Ru}(0001) .^{21}$ This can be used as a model system to reach an understanding of the intercalation process, without concerns arising from the pre-existence of a significant number of defects. We find that heteroatoms, graphene, and substrate need to be considered as a whole in order to understand the intercalation process. Our results may have impact for the large area production of devices based on hybrids between graphene and hetero-materials layers, which may be challenging to achieve otherwise. ${ }^{19,20}$ Other combinations of heteroatoms (such as $\mathrm{Ni}, \mathrm{Pd}$ and $\mathrm{Pt}$ ) and substrates (such as $\operatorname{Ir}(111)$ and $\mathrm{SiC}(0001)$ ) are also investigated to support the generality of our study.

\section{EXPERIMENTAL SECTION}

Intercalation of Si. Single layer graphene (SLG) is grown by exposing a $\mathrm{Ru}(0001)$ single crystal surface to ethylene at $1300 \mathrm{~K}^{21}$ The process is performed in an Omicron low-temperature scanning tunneling microscope (STM) with a base pressure lower than $\times 10^{-10}$ mbar. A current is applied to the surface of a thin Si wafer to sublimate $\mathrm{Si}$ atoms onto the SLG substrate, kept at room temperature. Calibration is done by measuring $\mathrm{Si}$ deposition onto a clean $\mathrm{Ru}(0001)$ surface, where $\mathrm{Si}$ can form monolayer (ML) islands. Based on STM images of different areas of the sample, a statistic analysis is done to determine the average Si coverage on $\mathrm{Ru}(0001)$. The heating current is fixed, and the the amount of $\mathrm{Si}$ deposited is assumed proportional to the evaporation time. Different Si coverages can be achieved by carefully adjusting the evaporation time. The calibration process is repeated more than 10 times to ensure accuracy. The sample is then annealed at different temperatures to initiate the intercalation process.

$\mathrm{Ar}^{+}$Ion Bombardment. An Omicron ISE 10 ion source is used to create single vacancy defects on SLG. The density of defects is determined by ion energy, Ar base pressure, and filament emission

Received: November 23, 2014

Published: May 11, 2015 
current, as well as bombardment time. We fix the ion energy at $100 \mathrm{eV}$, the Ar pressure at $3 \times 10^{-6} \mathrm{mbar}$, and the emission current at $0.1 \mathrm{~mA}$ and vary the bombardment time to tune the density of defects.

DFT Calculations. We use the local density approximation ${ }^{22}$ within the Vienna ab initio simulation package, ${ }^{23}$ and the projector augmented wave $(\mathrm{PAW})^{24}$ method. More details are provided in the Supporting Information.

\section{RESULTS AND DISCUSSION}

Figure 1a plots a $90 \times 90 \mathrm{~nm}$ STM topographic image of graphene after partial intercalation of Si. Before Si intercalation,

(a)

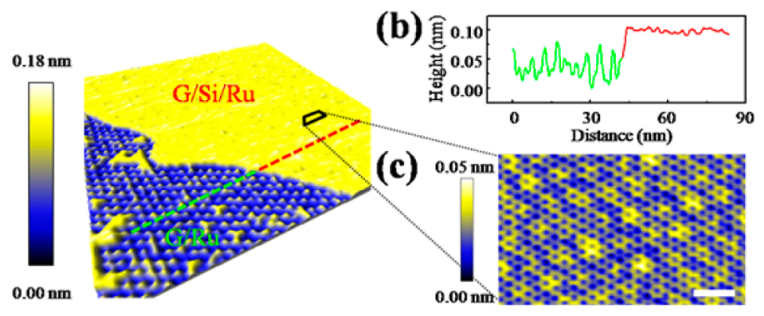

(d)

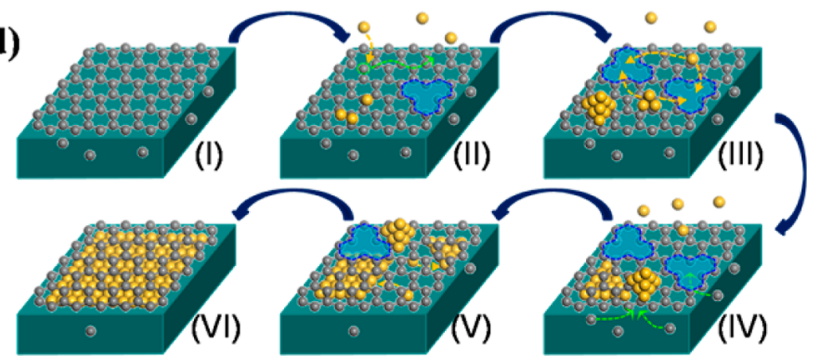

Figure 1. STM topography of SLG/Si/Ru and proposed intercalation process. (a) Three-dimensional view of the intercalated Si layer. (b) Height profile along the line depicted in (a). (c) Atomic resolution image showing the SLG lattice on top of the intercalated Si layer in the rectangular area highlighted in (a). The scale bar is $1 \mathrm{~nm}$. (d) Schematic intercalation process. The yellow and green arrows represent diffusion paths for the $\mathrm{Si}$ and $\mathrm{C}$ atoms, respectively.

intact SLG can be seen (see Supporting Information Figure S1). ${ }^{21}$ Figure 1a shows a smooth and flat surface that can be assigned as SLG/Si/Ru with a Si ML between the SLG and Ru. The rest of the image is a region with a small percentage of $\mathrm{Si}$, as evidenced by the appearance of small isolated $\mathrm{Si}$ islands underneath graphene, with a line profile shown in Figure $1 \mathrm{~b}$. The high-resolution image in Figure 1c further reveals that the SLG is intact and uniform after $\mathrm{Si}$ intercalation, which is confirmed by both STM characterization on different sample locations and Raman spectroscopy ${ }^{25}$ (see Supporting Information Figure S2).

A few mechanisms have been proposed to account for similar intercalation processes. ${ }^{71,114,16,18}$ Heteroatoms can diffuse through graphene island edges or pre-existing defects $7,11,14,18$ or exchange with carbon atoms. ${ }^{16}$ However, these mechanisms do not explain our observations, since our SLG has negligible pre-existing defects. ${ }^{21}$ Previous low-energy electron microscopy studies ${ }^{16}$ suggested that the $\mathrm{Si}$ atoms could directly penetrate through SLG without requirement of edges ${ }^{14}$ or wrinkles. ${ }^{18}$ But, the temperature in our experiments is not enough to break the carbon-carbon bond (the bond energy was estimated to be $\sim 5.67 \mathrm{eV},{ }^{26}$ corresponding to a temperature of $\sim 6.6 \times 10^{4} \mathrm{~K}$ ) or to enable the $\mathrm{Si}-\mathrm{C}$ exchange process, requiring at least 3 $\mathrm{eV}^{16}$
Figure 1d outlines our proposed mechanism by highlighting a few key steps: cooperative creation of defects (stage II), migration of heteroatoms through graphene to the $\mathrm{Ru}$ surface (stage III), self-repair of the graphene lattice (stage IV), and assembly of heteroatoms to form an intercalated layer (stage V). These four steps are intercorrelated and can happen simultaneously, which makes it challenging for unambiguous characterization.

Figure 2a shows a typical image after deposition of $0.05 \mathrm{ML}$ Si onto a SLG/Ru surface, followed by annealing at $400{ }^{\circ} \mathrm{C}$ for

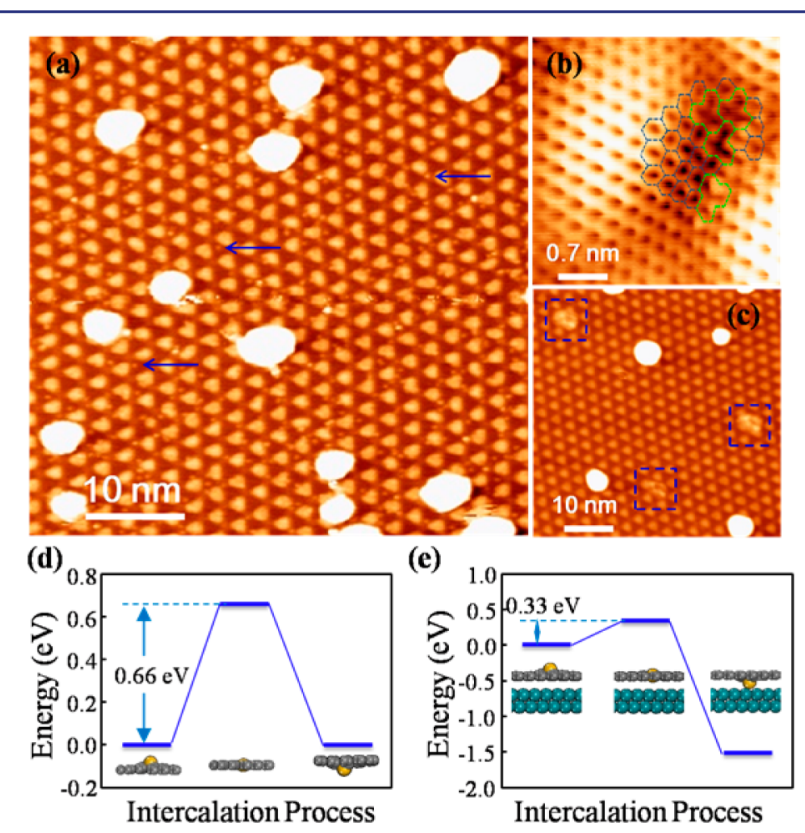

Figure 2. Si-induced defect formation. (a) STM image of SLG/Ru with predeposition of $0.05 \mathrm{ML} \mathrm{Si}$ at room temperature, followed by thermal annealing at $400{ }^{\circ} \mathrm{C}$ for $10 \mathrm{~min}$. (b) Atomically resolved image revealing a carbon vacancy (bias voltage $V_{\mathrm{s}}=-0.1 \mathrm{~V}$, tunneling current $I_{\mathrm{t}}=0.3 \mathrm{nA}$ ). (c) STM image of the sample in (a) after another thermal annealing at $450{ }^{\circ} \mathrm{C}$ for $30 \mathrm{~min}$. (d and e) Calculated energy barrier of $\mathrm{Si}$ intercalation without and with $\mathrm{Ru}$ substrate, respectively.

$10 \mathrm{~min}$, while Figure S3 in the Supporting Information plots the images for lower annealing temperatures. In addition to large bright protrusions, assigned to as-deposited Si clusters, many randomly distributed small dot-like features can be seen (blue solid arrows in Figure 2a, see also Supporting Information Figure S4a,b). These features can be assigned to carbon vacancies accompanied by distortion of the honeycomb lattice, as revealed in the high-resolution image of Figure 2b. Another evidence of our assignment to carbon vacancies is that the small dot-like features in Figure 2a can only be observed at 350-400 ${ }^{\circ} \mathrm{C}$ within a short time period $(\sim 10 \mathrm{~min})$. At higher temperatures and/or for longer times $(>30 \mathrm{~min}) \mathrm{Si}$ adatoms can migrate, accumulate, and combine with carbon vacancies, resulting in small bumps with different apparent height (e.g., dashed squares in Figure 2c). To confirm this, we perform a control experiment by heating graphene on the $\mathrm{Ru}$ substrate under the same conditions, but without predeposition of Si. In this case we do not detect any vacancy features in Figure 2 (see also Supporting Information Figure S4c). This suggests that the involvement of $\mathrm{Si}$ is a prerequisite for defect formation under our experimental conditions.

We further compare the defect formation energies in four different scenarios (as listed in Supporting Information Figure 
S5) by ab initio calculations: ${ }^{27}$ free-standing SLG, SLG/Ru, free-standing SLG with a Si adatom, and SLG/Ru with a Si adatom. An energy reduction from $8.09 \mathrm{eV}$ (free-standing SLG) to $0.23 \mathrm{eV}$ (Si-SLG-Ru) is observed after inclusion of interactions between $\mathrm{Si}$ adatoms, graphene, and substrate, making it possible to create vacancies in our experimental conditions.

The availability of carbon vacancies can thus facilitate migration of Si atoms through SLG. In order to evaluate the role of defects, we also perform a control experiment by creating single vacancy defects by low-energy ion bombardment. ${ }^{28}$ Argon ions with an energy of $100 \mathrm{eV}$ can create single vacancy defects on SLG/Ru, with defect density controllable by changing the dosage and duration (Supporting Information Figures S6 and S7). The same amount (2 ML) of Si is deposited onto SLG/Ru samples with different initial defect density (Supporting Information Figure S6a-c), followed by annealing at $700{ }^{\circ} \mathrm{C}$ for $30 \mathrm{~min}$. The higher the initial defect density, the more $\mathrm{Si}$ can be intercalated (Supporting Information Figure S6d-f), suggesting that defects indeed enable and promote intercalation, supporting the proposed defect assisted intercalation process.

In order to evaluate the role of the substrate in the intercalation process, we employ the climb nudged elastic band method $^{29}$ to simulate migration of a single $\mathrm{Si}$ atom through a vacancy. Figure $2 \mathrm{~d}$,e compares the energy barrier difference for the migration process without and with consideration of the effect of $\mathrm{Ru}(0001)$. We observe a significant reduction of the barrier from $0.66 \mathrm{eV}$ (without substrate) to $0.33 \mathrm{eV}$ (with substrate), suggesting that the $\mathrm{Ru}$ substrate can further reduce the energy barrier of this process, as well as the total energy of the system, during Si intercalation. This effect also makes the inverse process (i.e., migration of $\mathrm{Si}$ atoms from the interface to the upper SLG surface) less likely under our experimental conditions.

We observe that the honeycomb carbon lattice can be restored after $\mathrm{Si}$ intercalation, with the removal of vacancies in the temperature range $300-800{ }^{\circ} \mathrm{C}$. In order to remove vacancies, extra carbon atoms are required. These can originate either from the knockout carbon atoms in the defect formation stage or from the bulk $\mathrm{Ru}$ substrate. ${ }^{30}$ To confirm this, we investigate the relation between the SLG defect density and the annealing temperature. Figure $3 \mathrm{a}-\mathrm{c}$ shows three STM images of SLG/Ru after annealing at 25,300 , and $450{ }^{\circ} \mathrm{C}$, respectively. Figure $3 \mathrm{~d}$ indicates that the defect density substantially decreases with the increase in temperature. Annealing at 700 ${ }^{\circ} \mathrm{C}$ for $30 \mathrm{~min}$ is enough to repair almost all defects on the SLG surface after $\mathrm{Si}$ intercalation. This process may be critical in order to create high-quality graphene-based hybrid structures.

We now explore the evolution and assembly of the intercalated Si layer (i.e., stages V and VI in Figure 1d). Reference 31 suggested that the intercalated atoms could diffuse into the metal substrate and form alloys. We observe that the $\mathrm{Si}$ atoms tend to spread at the interface without the formation of alloys. Figure $4 a-c$ shows the development of three different structures with increasing $\mathrm{Si}$ adatoms from 0.3 to $0.8 \mathrm{ML}$. For low coverage, the intercalated $\mathrm{Si}$ atoms preferentially migrate underneath the graphene's atop sites ${ }^{32}$ (hills of the moiré structure) (Figure 4a). This is supported by the fact that the intercalated atop sites become enlarged, with a topographic distortion from the usual round shape (Supporting Information Figure S1). Once all available atop sites are occupied, more incoming $\mathrm{Si}$ atoms start occupying face-
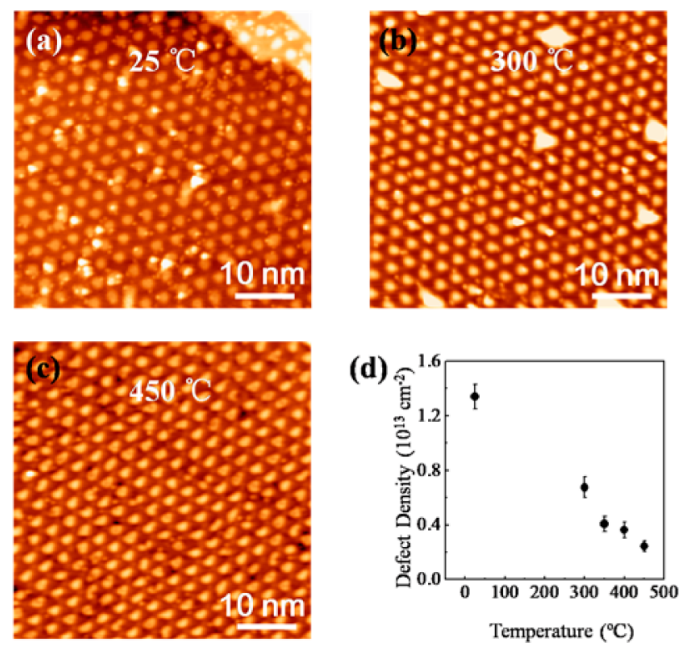

Figure 3. Removal of vacancies on SLG/Ru. STM images of the SLG/ Ru samples annealed at (a) $25{ }^{\circ} \mathrm{C}$, (b) $300{ }^{\circ} \mathrm{C}$, and (c) $450{ }^{\circ} \mathrm{C}$ for 30 $\mathrm{min}$. These samples have the same initial defect density, controlled by argon ion bombardment. (d) Relation between defect density on the $\mathrm{SLG} / \mathrm{Ru}$ surface and annealing temperature. Each data point is obtained by averaging eight $50 \times 50 \mathrm{~nm}$ STM images taken on different sample areas for a given temperature.

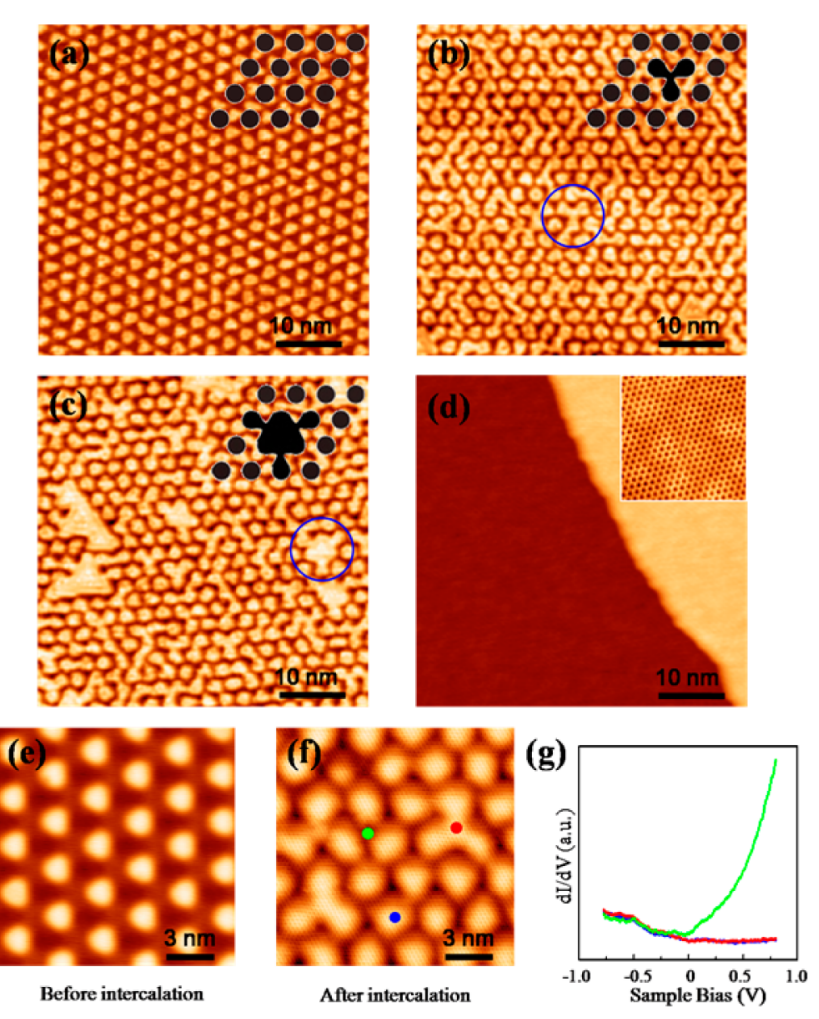

Figure 4. Evolution of the intercalated Si layer between SLG and the $\mathrm{Ru}$ substrate.The intercalated $\mathrm{Si}$ atoms can sequentially occupy the areas below (a) atop sites, (b) fcc sites, and (c) hcp sites. The inset shows the corresponding structural configuration of the intercalated $\mathrm{Si}$ atoms. (d) A full ML Si is formed at the interface. Inset is a $4.5 \times 4.5$ $\mathrm{nm}$ atomic resolution image showing the SLG lattice after intercalation. (e and f) Typical topography $\left(V_{s}=-0.05 \mathrm{~V}, I_{\mathrm{t}}=1.0\right.$ $\mathrm{nA}$ ) before and after $\mathrm{Si}$ intercalation. $(\mathrm{g}) \mathrm{d} I / \mathrm{d} V$ spectra acquired at the different locations marked in (f). The red, blue, and green curves correspond to the $\mathrm{d} I / \mathrm{d} V$ spectra acquired at the intercalated fcc sites, the intercalated atop sites, and the non-intercalated fcc sites, respectively. 
centered cubic (fcc, medium regions of the moiré structure) and then hexagonal close packed (hcp, lowest regions of the moire structure) sites, leading to the appearance of Y-shaped and triangular structures, as for Figures $4 b, c$, respectively. Once the atop, fcc, and hcp sites are fully occupied, a full Si ML underneath graphene is completed (Figure 4d), with the manifestation of different structural and electronic characteristics. For example, a variation of topographic features before and after $\mathrm{Si}$ intercalation can be found by comparing Figure $4 \mathrm{e}$ with $4 f$. The $d I / d V$ curves measured at the intercalated fcc and atop sites show similar features, but differ from those of the non-intercalated fcc sites (Figures $4 \mathrm{f}, \mathrm{g}$ ). This can be understood, since graphene becomes electronically decoupled from the substrate after $\mathrm{Si}$ intercalation.

We then perform similar experiments as well as DFT calculations to evaluate and compare different combinations of heteroatoms and substrates, with a few examples highlighted in Figure 5. Our calculations show that different systems can

(a)
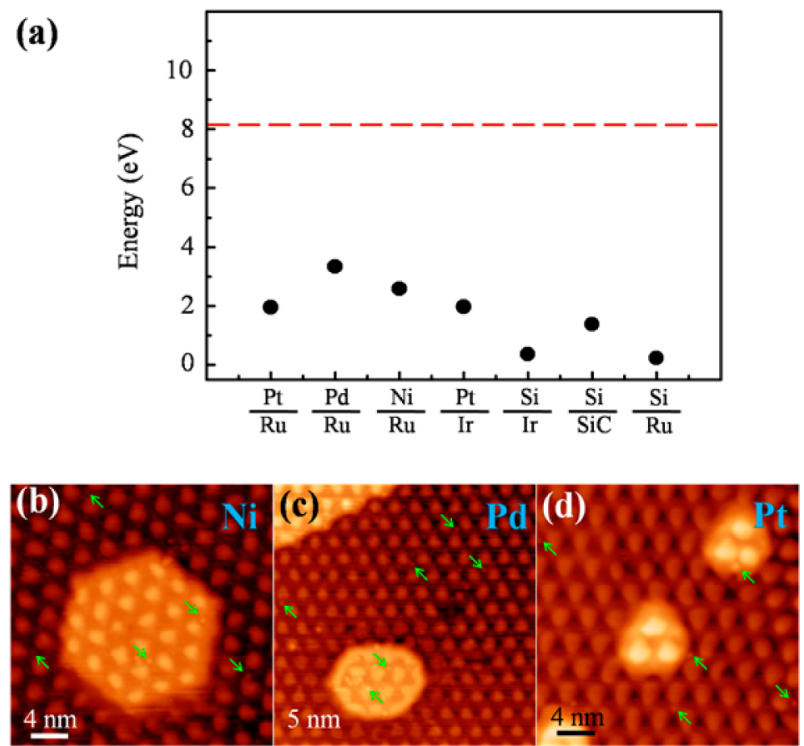

Figure 5. (a) Calculated formation energy for single vacancies in different systems. The red dashed line indicates the defect formation energy in free-standing SLG, and the black dots represent the corresponding energies in different systems. (b-d) STM images showing the SLG/Ru surface after intercalation of Ni, Pd, and Pt. The green arrows highlight the defects created in the intercalation process.

manifest similar defect opening processes with the reduction of the defect formation energy (Figure 5a), highlighting the generality of our proposed mechanism, i.e., carbon vacancies created by the interaction between heteroatoms, graphene, and substrate enable the heteroatoms to migrate through the defect sites and form an ordered intercalated ML, while defects are then removed, restoring the SLG crystalline lattice. Figure $5 b-$ $\mathrm{d}$ shows experimental confirmation of the process in the case of $\mathrm{Ni}, \mathrm{Pd}, \mathrm{Pt}$ intercalation between SLG and $\mathrm{Ru}$ (green arrows in Figure $5 b-d)$. These are similar to those discussed above for the $\mathrm{Si}$ intercalation process.

\section{CONCLUSIONS}

We studied the intercalation process of heteroatoms between a graphene layer and a metal substrate. This is governed by the interplay between heteroatoms, graphene, and substrate. This cooperative intercalation can result in both the formation of large-scale intercalated layers and the restoration of the graphene lattice at relatively low temperatures. This makes it possible to create vertical graphene-based heterostructures, minimizing the interfacial roughness. This may be critical for the future development of mass-scale devices based on heterostructures.

\section{ASSOCIATED CONTENT}

\section{S Supporting Information}

Information and figures regarding sample preparation and characterization; detailed discussions of DFT calculations and $\mathrm{Ar}^{+}$ion bombardment. The Supporting Information is available free of charge on the ACS Publications website at DOI: $10.1021 / \mathrm{ja} 5113657$.

\section{AUTHOR INFORMATION}

\section{Corresponding Authors}

*acf26@eng.cam.ac.uk

*hjgao@iphy.ac.cn

\section{Author Contributions}

\#These authors contributed equally.

Notes

The authors declare no competing financial interest.

\section{ACKNOWLEDGMENTS}

We acknowledge funding from the National Science Foundation of China, National "973" project of China, the Chinese Academy of Sciences, SSC, ONR (award no. N000141410328), NSF (DMR1307800), the EU Graphene Flagship (contract no. CNECT-ICT-604391), a Royal Society Wolfson Research Merit Award, the European Research Council Grants NANOPOTS, Hetero2D, EU grants GENIUS, MEM4WIN, CareRAMM, EPSRC grants EP/K01711X/1, EP/ K017144/1.

\section{REFERENCES}

(1) Ferrari, A. C.; et al. Nanoscale 2015, 7, 4598-4810.

(2) Novoselov, K. S.; Geim, A. K.; Morozov, S. V.; Jiang, D.; Katsenlson, M. I.; Grigorieva, I. V.; Dubonos, S. V.; Firsov, A. A. Nature 2005, 438, 197-200.

(3) Wu, Y. Q.; Jenkins, K. A.; Valdes-Garcia, A.; Farmer, D. B.; Zhu, Y.; Bol, A. A.; Dimitrakopoulos, C.; Zhu, W.; Xia, F.; Avouris, P.; Lin, Y. M. Nano Lett. 2012, 12, 3062-3067.

(4) Bonaccorso, F.; Sun, Z.; Hasan, T.; Ferrari, A. C. Nat. Photonics 2010, 4, 611-622.

(5) Enderlein, C.; Kim, Y. S.; Bostwick, A.; Rotenberg, E.; Horn, K. New J. Phys. 2010, 12, 033014.

(6) Virojanadara, C.; Watcharinyanon, S.; Zakharov, A. A.; Johansson, L. I. Phys. Rev. B 2010, 82, 205402.

(7) Jin, L.; Fu, Q.; Mu, R.; Tan, D.; Bao, X. Phys. Chem. Chem. Phys. 2011, 13, 16655-16660.

(8) Sicot, M.; Leicht, P.; Zusan, A.; Bouvron, S.; Zander, O.; Weser, M.; Dedkov, Y. S.; Horn, K.; Fonin, M. ACS Nano 2012, 6, 151-158.

(9) Huang, L.; Pan, Y.; Pan, L.; Gao, M.; Xu, W.; Que, Y.; Zhou, H.; Wang, Y.; Du, S.; Gao, H. J. Appl. Phys. Lett. 2011, 99, 163107.

(10) Mao, J. H.; Huang, L.; Pan, Y.; Gao, M.; He, J.; Zhou, H.; Guo, H.; Tian, Y.; Zou, Q.; Zhang, L.; Zhang, H.; Wang, Y.; Du, S.; Zhou, X.; Castro Neto, A. H.; Gao, H. J. Appl. Phys. Lett. 2012, 100, 093101.

(11) Xia, C.; Watcharinyanon, S.; Zakharov, A. A.; Yakimova, R.; Hultman, L.; Johansson, L. I.; Virojanadara, C. Phys. Rev. B 2012, 85, 045418.

(12) Bonaccorso, F.; Lombardo, A.; Hasan, T.; Sun, Z.; Colombo, L.; Ferrari, A. C. Mater. Today 2012, 15, 564-589.

(13) Riedl, C.; Coletti, C.; Iwasaki, T.; Zakharov, A. A.; Starke, U. Phys. Rev. Lett. 2009, 103, 246804. 
(14) Sutter, P.; Sadowski, J. T.; Sutter, E. A. J. Am. Chem. Soc. 2010, 132, 8175-8179.

(15) Feng, X. F.; Maier, S.; Salmeron, M. J. Am. Chem. Soc. 2012, 134, $5662-5668$.

(16) Cui, Y.; Gao, J.; Jin, L.; Zhao, J.; Tan, D.; Fu, Q.; Bao, X. Nano Res. 2012, 5, 352-360.

(17) Zhao, W.; Tan, P. H.; Liu, J.; Ferrari, A. C. J. Am. Chem. Soc. 2011, 133, 5941-5946.

(18) Petrović, M.; Šrut Rakić, I.; Runte, S.; Busse, C.; Sadowski, J. T.; Lazić, P.; Pletikosić, I.; Pan, Z.-H.; Milun, M.; Pervan, P.; Atodiresei, N.; Brako, R. Šokčević, D.; Valla, T.; Michely, T.; Kralj, M. Nat. Commun. 2013, 4, 2772.

(19) Britnell, L.; Gorbachev, R. V.; Jalil, R.; Belle, B. D.; Schedin, F.; Mishchenko, A.; Georgiou, T.; Katsnelson, M. I.; Eaves, L.; Morozov, S. V.; Peres, N. M. R.; Leist, J.; Geim, A. K.; Novoselov, K. S.; Ponomarenko, L. A. Science 2012, 335, 947-950.

(20) Haigh, S. J.; Gholinia, A.; Jalil, R.; Romani, S.; Britnell, L.; Elias, D. C.; Novoselov, K. S.; Ponomarenko, L. A.; Geim, A. K.; Gorbachev, R. Nat. Mater. 2012, 11, 764-767.

(21) Pan, Y.; Zhang, H.; Shi, D.; Sun, J.; Du, S.; Liu, F.; Gao, H. J. Adv. Mater. 2009, 21, 2777-2780.

(22) Perdew, J. P.; Zunger, A. Phys. Rev. B 1981, 23, 5048-5079.

(23) Kresse, G.; Furthmuller, J. Phys. Rev. B 1996, 54, 11169-11186.

(24) Blochl, P. E. Phys. Rev. B 1994, 50, 17953-17979.

(25) Ferrari, A. C.; Basko, D. M. Nat. Nanotechnol. 2013, 8, 235-246.

(26) Girit, Ç. Ö.; Meyer, J. C.; Erni, R.; Rossell, M. D.; Kisielowski, C.; Yang, L.; Park, C.-H.; Crommie, M. F.; Cohen, M. L.; Louie, S. G.; Zettl, A. Science 2009, 323, 1705-1708.

(27) Wang, L.; Zhang, X.; Chan, H. L. W.; Yan, F.; Ding, F. J. Am. Chem. Soc. 2013, 135, 4476-4482.

(28) Ugeda, M. M.; Brihuega, I.; Guinea, F.; Gómez-Rodríguez, J. M. Phys. Rev. Lett. 2010, 104, 096804.

(29) Henkelman, G.; Uberuaga, B. P.; Jonsson, H. A. J. Chem. Phys. 2000, 113, 9901-9904.

(30) Cui, Y.; Fu, Q.; Bao, X. Phys. Chem. Chem. Phys. 2010, 12, 5053-5057.

(31) Vilkov, O.; Fedorov, A.; Usachov, D.; Yashina, L. V.; Generalov, A. V.; Borygina, K.; Verbitskiy, N. I.; Grüneis, A.; Vyalikh, D. V. Sci. Rep. 2013, 3, 2168.

(32) Zhou, H. T.; Mao, J. H.; Li, G.; Wang, Y. L.; Feng, X. L.; Du, S. X.; Mullen, K.; Gao, H. J. Appl. Phys. Lett. 2011, 99, 153101.

\section{NOTE ADDED AFTER ASAP PUBLICATION}

The uncorrected proof version was published ASAP on May 18, 2015. The final corrected version was published on May 29, 2015. 